\title{
Effects of Shift and Night Work in the Offshore Petroleum Industry: A Systematic Review
}

\author{
Ingrid Nesdal FOSSUM ${ }^{1 *}$, Bjørn BJORVATN ${ }^{2,3}$, Siri WAAGE $^{2,3}$ and Ståle PALLESEN PAL $^{1,3}$ \\ ${ }^{1}$ Department of Psychosocial Science, University of Bergen, Norway \\ ${ }^{2}$ Department of Global Public Health and Primary Care, University of Bergen, Norway \\ ${ }^{3}$ Norwegian Competence Center for Sleep Disorders, Haukeland University Hospital, Norway
}

Received March 14, 2013 and accepted May 31, 2013

Published online in J-STAGE June 26, 2013

\begin{abstract}
Shift and night work are associated with several negative outcomes. The aim of this study was to make a systematic review of all studies which examine effects of shift and night work in the offshore petroleum industry, to synthesize the knowledge of how shift work offshore may affect the workers. Searches for studies concerning effects on health, sleep, adaptation, safety, working conditions, family- and social life and turnover were conducted via the databases Web of Knowledge, PsycINFO and PubMed. Search was also conducted through inspection of reference lists of relevant literature. We identified studies describing effects of shift work in terms of sleep, adaptation and re-adaptation of circadian rhythms, health outcomes, safety and accidents, family and social life, and work perceptions. Twenty-nine studies were included. In conclusion, the longitudinal studies were generally consistent in showing that adaptation to night work was complete within one to two weeks of work, while re-adaptation to a daytime schedule was slower. Shift workers reported more sleep problems than day workers. The data regarding mental and physical health, family and social life, and accidents yielded inconsistent results, and were insufficient as a base for drawing general conclusions. More research in the field is warranted.
\end{abstract}

Key words: Accidents, Adaptation, Health, Shift work, sleep, Night work, Offshore, Petroleum industry

\section{Introduction}

Shift work is commonly defined as work that occurs between 1900 and 0600 hours $^{1)}$. Night work involves a type of shift work where the majority of the work takes place between 2200 and 0600 hours $^{2}$. Studies show that shift work has increased during the past decades, due among other things to the industry's demand for continuous production ${ }^{3)}$.

Shift work and night work are associated with a range of negative outcomes. The most common health-related

*To whom correspondence should be addressed. E-mail: ifo026@webmail.uib.no

(C)2013 National Institute of Occupational Safety and Health effect of shift work is disturbed sleep, such as difficulty falling asleep, and shortened sleep duration ${ }^{4)}$. Shift work is also associated with increased fatigue ${ }^{4}$. Shift work may lead to shift work disorder (SWD) which in the second edition of the International Classification of Sleep Disorders $^{5)}$ is recognized as a circadian rhythm sleep disorder characterized by symptoms such as excessive sleepiness and insomnia ${ }^{6}$. The prevalence of SWD varies across different studies, from $10 \%{ }^{7}$ to $38 \%{ }^{8}$. The findings from a review conducted in 2008 indicated that less than $3 \%$ of permanent night workers show complete adaptation of their melatonin rhythm to night work ${ }^{9)}$. Shift and night work have also been shown to significantly increase the risk for work-related accidents ${ }^{10)}$.

Several other health issues are also related to shift work ${ }^{4)}$. 
A meta-analysis demonstrated that shift work increased the risk for cardiovascular disease by $40 \%{ }^{11)}$, and in a recent review, the authors concluded that shift workers appear to have increased risk of gastrointestinal symptoms, along with increased risk of peptic ulcer disease ${ }^{12)}$. A consistent finding is that prolonged night work is associated with an increased risk of breast cancer among women ${ }^{13,14)}$. Studies have further indicated an association between shift work and metabolic disturbances ${ }^{15-17)}$, and shift work is also found to be a risk factor for poor mental health ${ }^{18,19)}$. Shift work may also be detrimental for reproductive health, in terms of prolonged waiting time to pregnancy, low birth weight, increased risk for preterm delivery and increased risk for spontaneous abortions $^{20,21)}$. Shift work may also pose an increased risk for work-family conflicts ${ }^{22}$.

The offshore petroleum industry is characterized by an exceptionally high prevalence of shift work. A recent survey showed that approximately $55 \%$ of offshore workers in the Norwegian petroleum sector sometimes or regularly work night shifts ${ }^{23)}$. The standard offshore shift duration is 12 hours $^{24)}$, which is considerably longer than regular shifts onshore. Further, the offshore petroleum industry operates a number of different work schedules, with variations in duration of work and onshore leave periods ${ }^{25}$. While the most frequent arrangement in the UK petroleum offshore industry consists of two weeks of work offshore followed by two weeks at home ${ }^{26}$, most shift workers in the Norwegian sector follow a schedule consisting of two weeks of work offshore and four weeks off ${ }^{6}$. . The most common alternatives for shift rotation schedules are fixed shifts of 14 consecutive day shifts (14D) or 14 consecutive night shifts $(14 \mathrm{~N})$ alternating on tours, or swing shifts comprising one week of night shifts (normally the first one) and one week of day shifts within the same work period $(7 \mathrm{~N} / 7 \mathrm{D})^{27}$. The offshore industry further differs from the onshore sector as the former environment may be better adapted to 24 hour operations ${ }^{28)}$. Several factors influencing diurnal rhythms onshore, such as domestic duties and light exposure differ significantly in the isolated work environment offshore ${ }^{29}$. While offshore, the workers are isolated from family, relatives and friends; and have no commitments to daily family life or other onshore obligations ${ }^{29,30)}$. The offshore period involves long working hours, for most workers indoors, with little exposure to sunlight ${ }^{29)}$. Strict medical standards and safety courses are required to work offshore, and medical examinations given before recruitment and at regular intervals thereafter ensure that the personnel are physically and mentally fit for the harsh demands of the working environment ${ }^{31,32)}$.

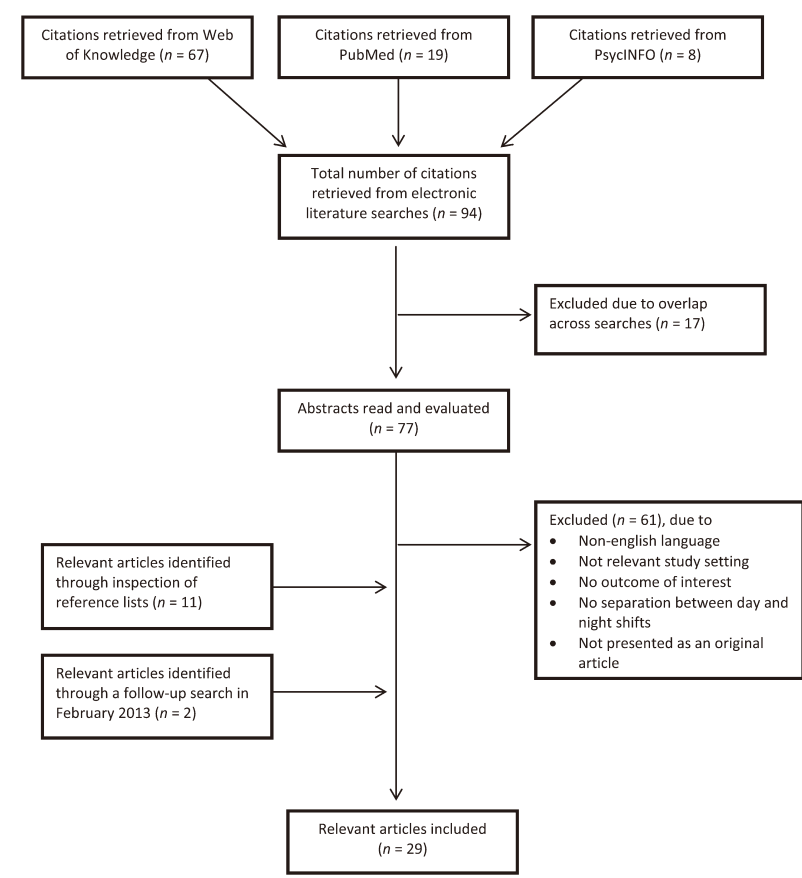

Fig. 1. Literature search and selection.

Hence, offshore personnel represent a selected group of particularly healthy and robust workers ${ }^{27,31)}$.

The different working conditions and shift work schedules make it difficult to generalize findings regarding effects of shift work onshore, where most of the shift work research has been conducted, to the offshore petroleum industry. Against this backdrop, we decided to conduct a systematic review of all studies concerning effects of shift work and night work in the offshore petroleum industry on health, sleep, adaptation, safety, working conditions, family- and social life, and turnover, in order to make a synthesis of the knowledge of how shift work offshore may affect the workers.

\section{Methods}

A literature search was conducted during June 2012 by use of the literature bases Web of Knowledge, PsycINFO and PubMed (Fig. 1). Key words that were used included "shiftwork" "or "shift work*" or "nightwork" or "night work*" combined with "offshore" or "off shore" and "oil*" or "petroleum". No year restrictions were used. In total, the searches resulted in 77 hits. In addition, relevant studies were identified by inspection of reference lists of central publications. Studies had to fulfill the following inclusion criteria in order to be included in the present review: 1) The publication was written in English; 2) The 
study was conducted on subjects working shifts in the offshore petroleum industry, thus studies concerning e.g. offshore shipping or fleet vessels were excluded; 3) The study concerned consequences of working shift or night, and included relevant outcome data in terms of health, sleep, adaptation, re-adaptation, work-family spillover, working conditions, accidents, safety and turnover; 4) The study separated between day and night shifts; 5) The study was presented as an original article, and published in a peer-reviewed journal. A total of 29 studies were identified satisfying the inclusion criteria.

In the present review, we differentiated between longitudinal and cross-sectional studies. Studies that included two or more measurement points were defined as longitudinal, whereas cross-sectional studies employed only one measurement point for each participant. Some of the studies classified as longitudinal also included cross-sectional findings.

\section{Results}

A total of 29 studies were included in this review; 13 examined outcome data of circadian adaptation and 11 concerned sleep. Eight studies dealt with shift work and mental or physical health, while two studies focused on family-work relations. Seven studies addressed safety/accidents directly, or included reaction time test assessments relevant for safety. One study concerned work perceptions, and one study addressed perceived mastery of work. Of the 29 studies, 27 were conducted in the Norwegian or in the UK offshore sector, of which 24 were from the North Sea. Two studies stemmed from the Campos Basin in Brazil. All studies reviewed had samples consisting of mostly or exclusively males. The average age in the different samples ranged between 33 and 49 years. The oldest study was published in 1990; the latest in 2012. Table 1 shows the results from the 14 cross-sectional studies included, and data from the 15 longitudinal studies that satisfied the inclusion criteria are presented in Table 2.

\section{Shift work and sleep}

In total, 11 studies concerning shift work and its association with sleep and sleepiness were identified. Eight of these studies were cross-sectional, three were longitudinal. Five cross-sectional studies compared offshore day workers with offshore shift/night workers. Two studies showed that the shift/night workers reported more sleep problems than day workers ${ }^{28,33)}$. In another study, permanent day workers reported better sleep quality than all other shift schedules $^{31)}$. However, in that study the relationship between shift schedules and sleep quality disappeared when controlling for risk perception and safety climate. The findings from yet another study indicated that shift/night work, compared to day work, was associated with longer sleep duration ${ }^{34)}$. A separate study demonstrated that offshore workers reported poor sleep quality during the first and the last days at home, independent of shift pattern at work $^{35)}$.

Three of the cross-sectional studies regarding sleep and sleepiness compared offshore workers with onshore workers across different shift patterns. One study found that in both groups, sleep quality was best during the off work period as compared to day work and night work ${ }^{36)}$. Further, offshore workers reported better sleep quality during night work than day work, while onshore workers reported the opposite. In the same study, offshore workers reported longer sleep duration during night work and lower sleep quality during day work than onshore workers ${ }^{36)}$. In another study, offshore workers reported better sleep quality and longer sleep duration than the onshore workers ${ }^{37}$. In both groups, the reported sleep duration and sleep quality were highest during the off work period, intermediate during day work and lowest during night work ${ }^{37)}$. In the only study investigating the prevalence of SWD, 23.3\% of swing shift oil rig workers fulfilled the criteria for the disorder ${ }^{6)}$. These workers, as compared to non-SWD workers, reported lower sleep quality, more subjective health complaints and poorer coping. However, in this study the reports of shift workers without SWD were no different from the reports of day workers regarding sleep and sleepiness.

One longitudinal study assessed sleep during different shift schedules in offshore workers. The findings showed that sleep efficiency was higher during day shift compared to night shift and swing shift; sleep duration was shorter during swing shift than during night shift and day shift, and sleep quality was better during swing shift than during night shift and day shift for the first week of work ${ }^{38)}$. Another longitudinal study (based on the same sample) examined sleepiness across the same three shift schedules. Subjective sleepiness was highest in the beginning of night and swing shifts ${ }^{39)}$. During night shift it gradually decreased, while during swing shift there was an initial decrease until a new peak in sleepiness appeared right after swinging from night to day work in the middle of the work period. Sleepiness at home was higher following night shift compared to following swing shift. Further, one longitudinal study found that both day workers and swing 
shift workers (7N/7D) reported poorer sleep quality and more insomnia symptoms at the end of a two week work period than at the start ${ }^{40)}$. Further, at the end of the work period, there was a higher proportion of insomniacs among swing shift workers than among day workers.

In sum, the findings concerning sleep quality and sleep duration are somewhat inconsistent regarding whether day or night workers are better off ${ }^{28,31,34,36,37)}$. The majority of studies found that offshore day shift workers reported better sleep quality, longer sleep duration and fewer sleep problems such as difficulty falling and staying asleep, and fragmented sleep, than offshore night shift workers ${ }^{28,31,33,37)}$.

\section{Shift work, adaptation and re-adaptation}

A total of 13 studies concerning adaptation and readaptation, all longitudinal, were identified.

\section{Adaptation}

Five studies assessed circadian adaptation to night work by measuring the urinary 6-sulphatoxymelatonin acrophase (aMT6s). In three of these studies, the majority of or all workers were fully adapted to night work within a week, by phase delay ${ }^{41-43)}$. One study found that most subjects (14 of 16) were adapted to night work by the final days of a two or three week night shift ${ }^{24)}$. In a study with two study groups, subjects studied in March adapted to night work within a week by phase advance ${ }^{44)}$. Subjects in the other group, assessed in November, showed no acrophase adaptation.

One study assessing circadian adaptation to night shift using cortisol measurement demonstrated that regardless of shift schedule (14N or 7N/7D), cortisol adaptation to night work was complete within a week ${ }^{29)}$.

Four studies assessed adaptation by means of sleep and sleepiness questionnaires/diaries and actigraphy recordings. Two studies showed that adaptation was complete within a few days, indicated by rapid reduction in sleepiness during work hours ${ }^{39,45}$. In another study, measures of sleepiness and sleep gradually improved during night shift, and the workers reported that adaptation was complete within a few days ${ }^{46)}$. One study found that in terms of sleep measured by sleep diary and actigraphy recordings, adaptation to swing shift seemed more difficult than adaptation to day shift and/or night shift ${ }^{38)}$.

Overall, the main findings regarding adaptation of circadian rhythms show that most workers were fully adapted to night work within one ${ }^{29,39,41-43,45,46)}$ to two or three weeks ${ }^{24)}$.

Re-adaptation
Ten studies assessed re-adaptation from night work back to a daytime schedule at work or at home. Three studies assessed re-adaptation by objective methods, using aMT6s acrophase or cortisol rhythm. In one of these studies, six of the 19 subjects who adapted to night work, phase advanced when swinging from night work to day work, and these six subjects were fully re-adapted within a week ${ }^{43)}$. The remaining subjects in this study did not achieve re-adaptation; seven showed little phase change while six continued to phase delay. A comparable study also found large individual variations during re-adaptation; as a group they did not adapt back to a day-night rhythm, with some subjects showing phase delay, others phase advance, and many showing only small phase changes ${ }^{42}$. In a study measuring cortisol rhythm, the rhythms were not readapted to a day schedule one week after returning home from two weeks of night work ${ }^{29)}$. Following swing shift, however, the cortisol rhythms were readapted after one week at home ${ }^{29)}$.

By use of a sleep diary and questionnaire, one study found that re-adaptation was slower and more difficult than adaptation to night shift; subjective sleepiness was higher during the first re-adaptation week at home than during the first week of night work ${ }^{45)}$. Another study found that sleepiness increased during re-adaptation following night shifts, as compared to following day or swing shifts ${ }^{39)}$. In another study, subjects reported that re-adaptation back to daytime work was slower than the preceding adaptation to night work ${ }^{46)}$. A different study using a sleep diary and actigraphy recordings demonstrated that there were no differences in re-adaptation to a daytime schedule at home across different shift schedules in terms of sleep ${ }^{38)}$.

In sum, several studies included in this review showed that re-adaptation from night work back to a daytime schedule offshore or at home was slower than adaptation to night work ${ }^{29,42,45)}$.

Three offshore intervention studies regarding sleep, adaptation and re-adaptation have been conducted. Two placebo-controlled intervention studies demonstrated that bright light treatment had a positive effect on adaptation to night work and on re-adaptation back to a daytime schedule, as measured by subjective sleep and sleepiness ratings ${ }^{47,48)}$. The light treatment was especially effective during re-adaptation ${ }^{47)}$. In another intervention study where the subjects received light treatment and wore sunglasses at appropriate times, objective sleep duration and sleep efficiency were improved after treatment ${ }^{49)}$. However, subjective data in this study showed decreased sleep quality during light treatment. Also, measures of 
Table 1. Summary of cross-sectional studies

\begin{tabular}{|c|c|c|c|c|c|}
\hline Authors/ date & Study method & Sample & Research topic & Measures & Main results \\
\hline $\begin{array}{l}\text { Parkes } \\
(1999)^{33)}\end{array}$ & $\begin{array}{l}\text { Questionnaire administered } \\
\text { once. Day work compared } \\
\text { with rotating day/night shift } \\
\text { work. Duration of work } \\
\text { period two-three weeks, } 12 \\
\text { h shifts. }\end{array}$ & $\begin{array}{l}1320 \text { North Sea oil and gas } \\
\text { industry personnel, } 680 \text { day } \\
\text { workers, } 640 \text { shift workers. } \\
\text { Mean age } 38.9 \text { yr. Average } \\
\text { response rate across } 17 \\
\text { installations of } 83 \% \text { (UK). }\end{array}$ & $\begin{array}{l}\text { Health, sleep, injuries. } \\
\text { How offshore shift } \\
\text { work predicts sleep } \\
\text { and health outcomes, } \\
\text { controlling for } \\
\text { individual differences, } \\
\text { job type and work } \\
\text { perceptions. }\end{array}$ & $\begin{array}{l}\text { Psychosomatic com- } \\
\text { plaints (headaches, } \\
\text { musculoskeletal pain, } \\
\text { gastric problems } \\
\text { and sleep problems), } \\
\text { affective distress and } \\
\text { work-related injuries. }\end{array}$ & $\begin{array}{l}\text { Shift workers reported more sleep } \\
\text { problems, gastric problems, psycholog- } \\
\text { ical distress and work-related injuries } \\
\text { than day workers. Controlled for job } \\
\text { type and demographic variables, shift } \\
\text { work predicted gastric problems and } \\
\text { sleep complaints. With no control for } \\
\text { job type, shift work also predicted } \\
\text { mental health and injuries. Rotating } \\
\text { day/night shift workers had lower BMI } \\
\text { than day workers. }\end{array}$ \\
\hline $\begin{array}{l}\text { Menezes et al. } \\
(2004)^{28)}\end{array}$ & $\begin{array}{l}\text { Questionnaire administered } \\
\text { once. Comparison of fixed } \\
\text { daytime workers and shift/ } \\
\text { night workers. } 81 \% \text { worked } \\
\text { for two weeks with a } \\
\text { midtour change. Usually } 12 \\
\text { h shifts. }\end{array}$ & $\begin{array}{l}\text { Offshore oil and gas instal- } \\
\text { lation personnel in the } \\
\text { Campos Basin, Brazil. } 86 \\
\text { day workers, } 80 \text { males, } 6 \\
\text { females, mean age } 35.8 \text { yr. } \\
93 \text { shift/night workers, } 91 \\
\text { males, } 2 \text { females, mean age } \\
37.7 \text { yr. Response rate } 58 \% \\
\text { (Brazil). }\end{array}$ & $\begin{array}{l}\text { Sleep in offshore } \\
\text { workers. }\end{array}$ & $\begin{array}{l}\text { Sleep parameters } \\
\text { (sleep quality, sleep } \\
\text { symptoms, sleep dura- } \\
\text { tion etc.). }\end{array}$ & $\begin{array}{l}\text { The shift/night group more often } \\
\text { reported poor sleep quality, difficulties } \\
\text { in falling and staying asleep, long sleep } \\
\text { onset latency, short sleep duration, } \\
\text { irregular bed times and feeling tired } \\
\text { upon awakening. }\end{array}$ \\
\hline $\begin{array}{l}\text { Hope et al. } \\
(2010)^{31)}\end{array}$ & $\begin{array}{l}\text { Questionnaire administered } \\
\text { once. Comparison of five } \\
\text { shift patterns; permanent } \\
\text { days or nights, fixed shifts, } \\
\text { swing shifts (7N/7D, } \\
7 \mathrm{D} / 7 \mathrm{~N}) \text { and "varying shift". }\end{array}$ & $\begin{array}{l}9,601 \text { offshore workers in } \\
\text { the North Sea, } 90 \% \text { males, } \\
10 \% \text { females; most predom- } \\
\text { inantly between the ages of } \\
31 \text { and } 50 . \text { Response rate } \\
\text { across } 52 \text { installations of } \\
50 \% \text { (Norway). }\end{array}$ & $\begin{array}{l}\text { Relationship between } \\
\text { risk perception, safety } \\
\text { climate and sleep } \\
\text { quality. }\end{array}$ & $\begin{array}{l}\text { Sleep quality, risk } \\
\text { perception, and safety } \\
\text { climate. }\end{array}$ & $\begin{array}{l}\text { Permanent day shift workers reported } \\
\text { better sleep quality than all other shift } \\
\text { arrangements, but with small effect } \\
\text { sizes. Shift patterns only predicted } \\
\text { sleep quality in univariate analysis, but } \\
\text { not in the multivariate model where } \\
\text { risk perception and safety climate were } \\
\text { included. }\end{array}$ \\
\hline $\begin{array}{l}\text { Waage et al. } \\
(2010)^{34)}\end{array}$ & $\begin{array}{l}\text { Questionnaire distributed } \\
\text { during the first work day } \\
\text { in day workers (14D) } \\
\text { and swing shift workers } \\
\text { (7N/7D), } 12 \text { h shifts during } \\
\text { two weeks of work. }\end{array}$ & $\begin{array}{l}204 \text { oil rig shift workers in } \\
\text { the North Sea. } 7 \text { females, } \\
197 \text { males; mean age } 42.9 \\
\text { yr. } 103 \text { swing shift workers, } \\
96 \text { day workers. Response } \\
\text { rate } 79 \% \text { (Norway). }\end{array}$ & $\begin{array}{l}\text { Sleep and health dur- } \\
\text { ing offshore work. }\end{array}$ & $\begin{array}{l}\text { Sleep parameters, } \\
\text { circadian preference } \\
\text { and subjective health } \\
\text { complaints. }\end{array}$ & $\begin{array}{l}\text { Swing shift was associated with longer } \\
\text { sleep duration, compared to day shift. } \\
\text { For swing shift workers, sleep duration } \\
\text { was negatively associated with age. } \\
\text { Shift type, age and shift work exposure } \\
\text { time seemed not to affect shift work } \\
\text { tolerance. }\end{array}$ \\
\hline $\begin{array}{l}\text { Rodrigues et } \\
\text { al. }(2001)^{35)}\end{array}$ & $\begin{array}{l}\text { Interviews of offshore shift } \\
\text { workers (rotating, on call, } \\
\text { fixed days + call outs, fixed } \\
\text { day, evenings + nights, and } \\
\text { fixed nights) and data from } \\
\text { work accident registry were } \\
\text { included. Mainly } 12 \mathrm{~h} \text { shifts } \\
\text { were worked. }\end{array}$ & $\begin{array}{l}51 \text { male offshore shift } \\
\text { workers in the Campos } \\
\text { Basin, Brazil, mean age } \\
37.6 \text { yr (Brazil). }\end{array}$ & $\begin{array}{l}\text { Offshore shift work } \\
\text { impacts on social and } \\
\text { family life, accidents, } \\
\text { sleep quality. }\end{array}$ & $\begin{array}{l}\text { Sleep quality, shift } \\
\text { work impact on social } \\
\text { and family life, ac- } \\
\text { cidents. }\end{array}$ & $\begin{array}{l}\text { Offshore shift workers reported dif- } \\
\text { ficulties in reconciling offshore work } \\
\text { with family life, as well as poor sleep } \\
\text { the first and last days during work off } \\
\text { period independent of shift type. The } \\
\text { relative risk of accidents was } 51 \% \\
\text { higher between } 1801 \text { and } 0559 \text { hours, } \\
\text { compared to between } 0600 \text { and } 1800 \\
\text { hours. }\end{array}$ \\
\hline $\begin{array}{l}\text { Parkes } \\
(1994)^{36)}\end{array}$ & $\begin{array}{l}\text { Questionnaire administered } \\
\text { once. Comparison of off- } \\
\text { shore and onshore workers } \\
\text { across night work, day } \\
\text { work and off work period. } \\
\text { Duration of work period } \\
\text { offshore was two weeks } \\
\text { with } 12 \text { h shifts. }\end{array}$ & $\begin{array}{l}\text { Male oil industry personnel } \\
\text { from three oil platforms in } \\
\text { the North Sea. } 84 \text { offshore } \\
\text { workers, mean age } 40.9 \mathrm{yr} \\
\text { and } 88 \text { onshore workers, } \\
\text { mean age } 44.5 \text { yr. Response } \\
\text { rate }>90 \% \text { (UK). Sample as } \\
\text { for Parkes (1992). }\end{array}$ & $\begin{array}{l}\text { Sleep patterns across } \\
\text { day shift, night shift } \\
\text { and during off work } \\
\text { period. }\end{array}$ & $\begin{array}{l}\text { Self-reported sleep } \\
\text { duration and sleep } \\
\text { quality during different } \\
\text { shifts schedules (day } \\
\text { work, night work, off } \\
\text { work period). }\end{array}$ & $\begin{array}{l}\text { Offshore workers reported longer night } \\
\text { work sleep duration and lower day } \\
\text { work sleep quality compared to those } \\
\text { onshore. In both groups, sleep quality } \\
\text { was best during off work period. For } \\
\text { the offshore group night work sleep } \\
\text { quality was higher than day work sleep } \\
\text { quality, while the opposite was found } \\
\text { for onshore workers. For onshore } \\
\text { workers day work sleep duration was } \\
\text { longer than night work sleep duration. }\end{array}$ \\
\hline $\begin{array}{l}\text { Parkes } \\
(2002)^{37)}\end{array}$ & $\begin{array}{l}\text { Questionnaire adminis- } \\
\text { tered once. Two weeks } \\
\text { of } 12 \mathrm{~h} \text { shifts, fixed shifts } \\
(14 \mathrm{D}, 14 \mathrm{~N}) \text { or swing shift } \\
(7 \mathrm{D} / 7 \mathrm{~N}, 7 \mathrm{~N} / 7 \mathrm{D}) \text { offshore. } \\
\text { Workers with fast, interme- } \\
\text { diate or weekly rotations } \\
\text { onshore. }\end{array}$ & $\begin{array}{l}456 \text { male offshore person- } \\
\text { nel from } 11 \text { platforms in the } \\
\text { North Sea, mean age } 38.4 \\
\text { yr and } 330 \text { male onshore } \\
\text { workers, mean age } 42.6 \\
\text { yr (UK). }\end{array}$ & $\begin{array}{l}\text { Sleep pattern across } \\
\text { day shift, night shift } \\
\text { and during off work. }\end{array}$ & $\begin{array}{l}\text { Self-reported sleep } \\
\text { duration and sleep } \\
\text { quality for three differ- } \\
\text { ent shifts. }\end{array}$ & $\begin{array}{l}\text { Offshore workers reported better sleep } \\
\text { quality and longer sleep duration than } \\
\text { onshore workers. For both offshore } \\
\text { and onshore workers: sleep duration } \\
\text { off work }>\text { day work }>\text { night work, and } \\
\text { sleep quality off work }>\text { day work }> \\
\text { night work. }\end{array}$ \\
\hline
\end{tabular}


Table 1. continued

\begin{tabular}{|c|c|c|c|c|c|}
\hline Authors/ date & Study method & Sample & Research topic & Measures & Main results \\
\hline $\begin{array}{l}\text { Waage et al. } \\
(2009)^{6)}\end{array}$ & $\begin{array}{l}\text { Questionnaire administered } \\
\text { during the first day of a } \\
\text { two week working period } \\
\text { reflecting symptoms during } \\
\text { the previous four week } \\
\text { period off. Swing shift } \\
\text { workers (7N/7D) were } \\
\text { compared to day workers. } \\
\text { Shift duration was } 12 \mathrm{~h} \text {. }\end{array}$ & $\begin{array}{l}103 \text { oil rig swing shift } \\
\text { workers, mean age } 39.8 \text { yr, } \\
98 \text { males, } 5 \text { females. } 96 \text { day } \\
\text { workers, no information } \\
\text { about gender and age. North } \\
\text { Sea. Response rate } 78.8 \% \\
\text { (Norway). }\end{array}$ & $\begin{array}{l}\text { Shift work disorder } \\
\text { (SWD) in offshore } \\
\text { workers. }\end{array}$ & $\begin{array}{l}\text { Questionnaires } \\
\text { about SWD, sleep, } \\
\text { sleepiness, insomnia, } \\
\text { circadian preference, } \\
\text { subjective health } \\
\text { complaints, psycho- } \\
\text { logical demands and } \\
\text { job control. }\end{array}$ & $\begin{array}{l}\text { SWD prevalence was } 23.3 \% \text {. Individu- } \\
\text { als with SWD reported poorer sleep } \\
\text { quality, more subjective health com- } \\
\text { plaints and poorer coping during the } \\
\text { four-weeks on leave compared to those } \\
\text { without SWD. Shift workers without } \\
\text { SWD were comparable to day workers } \\
\text { on all parameters but one; day workers } \\
\text { reported higher degree of job control. }\end{array}$ \\
\hline $\begin{array}{l}\text { Parkes } \\
(1992)^{32)}\end{array}$ & $\begin{array}{l}\text { Questionnaire administered } \\
\text { once. Comparison of swing } \\
\text { shift workers (7N/7D) off- } \\
\text { shore and onshore workers } \\
\text { with rapid rotation across } \\
\text { night shift, day shift and off } \\
\text { work period. Work period } \\
\text { offshore lasted for two } \\
\text { weeks with } 12 \mathrm{~h} \text { shifts. }\end{array}$ & $\begin{array}{l}\text { Male oil industry person- } \\
\text { nel in the North Sea. } 84 \\
\text { offshore workers, mean } \\
\text { age } 40.9 \text { yr and } 88 \text { onshore } \\
\text { workers, mean age } 44.5 \\
\text { yr. Response rate }>90 \% \text {. } \\
\text { Sample as for Parkes (1994) } \\
\text { (UK). }\end{array}$ & $\begin{array}{l}\text { Mental health among } \\
\text { offshore workers. }\end{array}$ & $\begin{array}{l}\text { Mental health and } \\
\text { neuroticism, work } \\
\text { condition measures. }\end{array}$ & $\begin{array}{l}\text { Offshore swing shift workers had } \\
\text { significantly higher anxiety scores. No } \\
\text { group differences regarding somatic } \\
\text { symptoms or social dysfunction. }\end{array}$ \\
\hline $\begin{array}{l}\text { Ljoså et al. } \\
(2011)^{50)}\end{array}$ & $\begin{array}{l}\text { Web-based question- } \\
\text { naire administered once. } \\
\text { Comparison of day work } \\
\text { and work involving night } \\
\text { shifts. Shifts lasted } 12 \mathrm{~h} \text { for } \\
\text { two weeks. }\end{array}$ & $\begin{array}{l}\text { 1,336 North Sea offshore } \\
\text { shift workers, } 83 \% \text { male, } \\
17 \% \text { female. Mean age } 45.1 \\
\text { yr. Shift schedule including } \\
\text { night work: } 680 \text { subjects. } \\
\text { Response rate } 56 \% \text {. Sample } \\
\text { as for Ljoså et al. (2012) } \\
\text { (Norway). }\end{array}$ & $\begin{array}{l}\text { Mental health among } \\
\text { offshore workers. }\end{array}$ & Mental distress. & $\begin{array}{l}\text { Shift schedules were only univariately } \\
\text { related to mental distress. Shift work } \\
\text { arrangements lost their significance } \\
\text { when adjusted for the individual fac- } \\
\text { tors of age, gender, marital status, and } \\
\text { shift work locus of control. }\end{array}$ \\
\hline $\begin{array}{l}\text { Parkes } \\
(2002)^{51)}\end{array}$ & $\begin{array}{l}\text { Questionnaire administered } \\
\text { once. Comparison of day } \\
\text { work with rotating day/ } \\
\text { night shift work offshore, } \\
\text { typically 7N/7D. Work } \\
\text { period of two weeks, } 12 \\
\text { h shifts. }\end{array}$ & $\begin{array}{l}1,574 \text { male offshore person- } \\
\text { nel in the North Sea, } 787 \\
\text { day-night shift workers and } \\
787 \text { day workers. Mean age } \\
38.7 \text { yr. Average response } \\
\text { rate across } 17 \text { installations: } \\
82.9 \% \text { (UK). }\end{array}$ & $\begin{array}{l}\text { Health, BMI. Interac- } \\
\text { tive effects of shift } \\
\text { work patterns, age and } \\
\text { years of shift work } \\
\text { exposure on BMI. }\end{array}$ & $\begin{array}{l}\text { Self-reported height } \\
\text { and weight. }\end{array}$ & $\begin{array}{l}\text { No main effect of shift pattern on BMI } \\
\text { itself, but continued exposure to rotat- } \\
\text { ing shift work was related to increased } \\
\text { BMI. Increase in BMI with age was } \\
\text { significantly greater for rotating shift } \\
\text { workers than for day workers. }\end{array}$ \\
\hline $\begin{array}{l}\text { Ljoså \& Lau } \\
(2009)^{52)}\end{array}$ & $\begin{array}{l}\text { Web-based question- } \\
\text { naire administered once. } \\
\text { Offshore: day work, fixed } \\
\text { shifts }(14 \mathrm{D}, 14 \mathrm{~N}), \text { swing } \\
\text { shifts }(7 \mathrm{D} / 7 \mathrm{~N}), \text { and "other } \\
\text { rotations". Onshore: all } \\
\text { types of shifts. }\end{array}$ & $\begin{array}{l}1,697 \text { onshore and offshore } \\
\text { petroleum personnel in the } \\
\text { Norwegian industry. } 299 \\
\text { females, } 1,398 \text { males, most } \\
\text { predominantly between the } \\
\text { ages of } 40 \text { and } 49 . \text { Response } \\
\text { rate } 55.9 \% \text { (Norway). }\end{array}$ & $\begin{array}{l}\text { Family and social life } \\
\text { in relation to shift rota- } \\
\text { tion patterns. }\end{array}$ & $\begin{array}{l}\text { Measures of satisfac- } \\
\text { tion with social and } \\
\text { family life and coping } \\
\text { strategies. }\end{array}$ & $\begin{array}{l}\text { Generally low scores on questions } \\
\text { concerning problems with social and } \\
\text { family life caused by shift work. The } \\
\text { majority of shift workers reported few } \\
\text { problems with social and domestic/ } \\
\text { family life, with onshore workers be- } \\
\text { ing worse off than offshore workers. }\end{array}$ \\
\hline $\begin{array}{l}\text { Parkes } \\
(2003)^{30)}\end{array}$ & $\begin{array}{l}\text { Questionnaire distributed } \\
\text { once. Comparison of shift } \\
\text { workers and day work- } \\
\text { ers onshore and offshore. } \\
\text { Offshore: } 12 \text { h shifts during } \\
\text { two week word periods } \\
\text { of fixed shifts }(14 \mathrm{D}, 14 \mathrm{~N}) \\
\text { or swing shifts }(7 \mathrm{D} / 7 \mathrm{~N} \text {, } \\
7 \mathrm{~N} / 7 \mathrm{D}) \text {. }\end{array}$ & $\begin{array}{l}1,867 \text { male oil industry } \\
\text { personnel in the North Sea. } \\
1,067 \text { offshore workers and } \\
800 \text { onshore workers. Mean } \\
\text { age for the whole sample } \\
\text { was } 41.1 \text { yr. Mean response } \\
\text { rate across } 17 \text { offshore } \\
\text { installations } 82.6 \% \text {, onshore } \\
\text { response rate on average } \\
65 \% \text { (UK). }\end{array}$ & $\begin{array}{l}\text { Work perceptions } \\
\text { among offshore and } \\
\text { onshore workers. }\end{array}$ & $\begin{array}{l}\text { Perceived work envi- } \\
\text { ronment. }\end{array}$ & $\begin{array}{l}\text { Offshore shift workers reported greater } \\
\text { exposure to physical stressors than } \\
\text { offshore day workers. The differences } \\
\text { in work perceptions were generally } \\
\text { more marked in the onshore setting, } \\
\text { where shift workers perceived their } \\
\text { environment less favorably than day } \\
\text { workers. }\end{array}$ \\
\hline $\begin{array}{l}\text { Ljoså et al. } \\
(2012)^{54)}\end{array}$ & $\begin{array}{l}\text { Web-based questionnaire } \\
\text { administered once. Com- } \\
\text { parison of day work and } \\
\text { work involving night shifts } \\
\text { during a two-week offshore } \\
\text { work period, } 12 \mathrm{~h} \text { shifts. }\end{array}$ & $\begin{array}{l}1,336 \text { offshore workers in } \\
\text { the North Sea. } 83 \% \text { males, } \\
17 \% \text { females. Mean age } \\
45.1 \text { yr. Response rate } \\
56 \% \text {. Comparison sample: } \\
\text { representative sample of } \\
\text { Scandinavian workers } \\
\text { (n=1,015). } 63 \% \text { males } \\
\text { and } 37 \% \text { females. Sample } \\
\text { as for Ljoså et al. (2011) } \\
\text { (Norway). }\end{array}$ & $\begin{array}{l}\text { Associations between } \\
\text { individual and work- } \\
\text { related factors and } \\
\text { perceived mastery of } \\
\text { work. }\end{array}$ & $\begin{array}{l}\text { Perceived mastery of } \\
\text { work, individual fac- } \\
\text { tors and work-related } \\
\text { factors. }\end{array}$ & $\begin{array}{l}\text { Night work was negatively related } \\
\text { to perceived mastery of work in a } \\
\text { bivariate analysis, but was unrelated to } \\
\text { perceived mastery of work in a multi- } \\
\text { variate analysis. Offshore shift workers } \\
\text { reported significantly higher levels of } \\
\text { perceived mastery of work than the } \\
\text { control group. }\end{array}$ \\
\hline
\end{tabular}

$\mathrm{D}=$ day (s); $\mathrm{N}=$ night $(\mathrm{s}) ; \mathrm{SWD}=$ shift work disorder. 
Table 2. Summary of longitudinal studies

\begin{tabular}{|c|c|c|c|c|c|}
\hline Authors/ date & Study method & Sample & Research topic & Measures & Main results \\
\hline $\begin{array}{l}\text { Saksvik et al. } \\
(2011)^{38)}\end{array}$ & $\begin{array}{l}\text { Daily sleep diary completion and } \\
\text { actigraphy recordings for one week } \\
\text { before work, during a two week work } \\
\text { period and one week following work } \\
\text { for three different work schedules } \\
(14 \mathrm{D}, 14 \mathrm{~N}, 7 \mathrm{~N} / \mathrm{D}) 12 \mathrm{~h} \text { shifts. }\end{array}$ & $\begin{array}{l}19 \text { oil installation } \\
\text { workers in the North } \\
\text { Sea, } 6 \text { females, } 13 \\
\text { males. Mean age } 44.4 \\
\text { yr. Response rate } \\
87.5 \% \text {. Sample as for } \\
\text { Harris } \text { et al. (2010) } \\
\text { and Waage } \text { et al. } \\
\text { (2012) (Norway). }\end{array}$ & $\begin{array}{l}\text { Adaptation } \\
\text { to shift work, } \\
\text { sleep during two } \\
\text { weeks of work } \\
\text { and re-adapta- } \\
\text { tion after the } \\
\text { work period. }\end{array}$ & $\begin{array}{l}\text { Subjective and } \\
\text { objective (ac- } \\
\text { tigraphy) sleep } \\
\text { measures. }\end{array}$ & $\begin{array}{l}\text { Sleep efficiency was higher during day work } \\
\text { than night work and swing shift. Sleep quality } \\
\text { was better during swing shift than during day } \\
\text { work and night work the first week of work. } \\
\text { Sleep duration was shorter during swing shift } \\
\text { than regular day work and night work. In terms } \\
\text { of sleep, adaptation to swing shift seemed } \\
\text { more difficult than adaptation to day work and } \\
\text { night work. Small differences in re-adaptation } \\
\text { between different shifts. }\end{array}$ \\
\hline $\begin{array}{l}\text { Waage et al. } \\
(2012)^{39)}\end{array}$ & $\begin{array}{l}\text { Participants were monitored for four } \\
\text { weeks; one before, two during and } \\
\text { one after the shift work. Assessment } \\
\text { was repeated for three different shift } \\
\text { schedules lasting for two weeks (14D, } \\
14 \mathrm{~N} \text {, or } 7 \mathrm{~N} / 7 \mathrm{D}) \text {. }\end{array}$ & $\begin{array}{l}19 \text { North Sea oil rig } \\
\text { workers ( } 6 \text { females, } \\
13 \text { males), mean age } \\
44.4 \text { yr. Response rate } \\
87.5 \% \text {. Sample as for } \\
\text { Harris et al. (2010) } \\
\text { and Saksvik et al. } \\
\text { (2011) (Norway). }\end{array}$ & $\begin{array}{l}\text { Sleepiness } \\
\text { during off- } \\
\text { shore work. } \\
\text { Adaptation to } \\
\text { night work and } \\
\text { re-adaptation } \\
\text { to daytime } \\
\text { schedule. }\end{array}$ & $\begin{array}{l}\text { Sleep and wake } \\
\text { diary completed } \\
\text { daily, reaction } \\
\text { time tests per- } \\
\text { formed six times } \\
\text { during each } \\
\text { work period. }\end{array}$ & $\begin{array}{l}\text { Sleepiness was highest during the first days } \\
\text { of swing and night shift, during night shift it } \\
\text { decreased. When swinging to day shift a new } \\
\text { peak in sleepiness was found during daytime. } \\
\text { Reaction time tests during the work period } \\
\text { showed no significant differences between } \\
\text { the shift schedules. There was a significantly } \\
\text { shorter reaction time the last day compared to } \\
\text { the beginning or middle of the work period. At } \\
\text { home, sleepiness was higher following night } \\
\text { shift compared to following swing shift. Ad- } \\
\text { aptation to night work happened within a few } \\
\text { days according to subjective measures. }\end{array}$ \\
\hline $\begin{array}{l}\text { Waage et al. } \\
(2012)^{40)}\end{array}$ & $\begin{array}{l}\text { Questionnaire administered twice } \\
\text { during a two week work period with } \\
12 \text { h shifts; during the first and last } \\
\text { workday. }\end{array}$ & $\begin{array}{l}\text { Oil rig workers } \\
\text { in the North Sea } \\
\text { working two weeks } \\
\text { of day shift ( } \mathrm{n}=90) \\
\text { or two weeks of a } \\
\text { swing shift schedule } \\
\text { (7N/7D) }(\mathrm{n}=93 \text { ). } 176 \\
\text { males and } 5 \text { females. } \\
\text { Mean age } 42.9 \text { yr. } \\
\text { Response rate } 72.6 \% \\
\text { (Norway). }\end{array}$ & $\begin{array}{l}\text { Subjective sleep } \\
\text { and subjective } \\
\text { health com- } \\
\text { plaints. }\end{array}$ & $\begin{array}{l}\text { Sleep and } \\
\text { insomnia } \\
\text { symptoms, } \\
\text { subjective health } \\
\text { complaints. }\end{array}$ & $\begin{array}{l}\text { Workers reported significantly poorer sleep } \\
\text { quality and more complaints of insomnia at } \\
\text { the end of the work period than at the start. No } \\
\text { significant difference in subjective health com- } \\
\text { plaints between the groups. No clear differ- } \\
\text { ences in changes in sleep quality, insomnia or } \\
\text { subjective health complaints during the work } \\
\text { period between day and swing shift workers. } \\
\text { Higher proportion of insomniacs among swing } \\
\text { shift workers than day workers at the end of } \\
\text { the work period. }\end{array}$ \\
\hline $\begin{array}{l}\text { Barnes et al. } \\
(1998)^{41)}\end{array}$ & $\begin{array}{l}\text { Urine samples collected several times } \\
\text { a day during two weeks of day shifts } \\
\text { and two weeks of night shifts. } 12 \mathrm{~h} \\
\text { shifts were worked. }\end{array}$ & $\begin{array}{l}11 \text { male subjects } \\
\text { studied in winter; six } \\
\text { maintenance workers } \\
\text { (mean age } 36.7 \mathrm{yr} \text { ) } \\
\text { and five drill crew } \\
\text { members (mean age } \\
45.0 \text { yr). } 23 \text { male } \\
\text { engineers studied in } \\
\text { summer, mean age } \\
37.5 \text { yr. North Sea } \\
\text { (UK). }\end{array}$ & $\begin{array}{l}\text { Adaptation to } \\
\text { night work. }\end{array}$ & $\begin{array}{l}\text { Changes in } \\
\text { 6-sulphatox- } \\
\text { ymelatonin } \\
\text { (aMT6s) acro- } \\
\text { phase. }\end{array}$ & $\begin{array}{l}\text { The subjects showed adaptation to night shift } \\
\text { by delay of the aMT } 6 \text { s rhythm within the first } \\
\text { week. No seasonal differences in adaptation } \\
\text { rates were found. }\end{array}$ \\
\hline $\begin{array}{l}\text { Gibbs et al. } \\
(2002)^{42)}\end{array}$ & $\begin{array}{l}\text { Urine samples collected several times } \\
\text { a day during two weeks of swing shift } \\
\text { (7N/7D), } 12 \mathrm{~h} \text { shift duration. }\end{array}$ & $\begin{array}{l}11 \text { male swing shift } \\
\text { workers from two oil } \\
\text { installations in the } \\
\text { North Sea, mean age } \\
44.7 \mathrm{yr} \text { (UK). }\end{array}$ & $\begin{array}{l}\text { Adaptation to } \\
\text { night work and } \\
\text { re-adaptation } \\
\text { back to day } \\
\text { work. }\end{array}$ & $\begin{array}{l}\text { Adaptation of } \\
\text { circadian phase, } \\
\text { assessed by } \\
\text { aMT6s acro- } \\
\text { phase. }\end{array}$ & $\begin{array}{l}8 \text { of } 11 \text { subjects adapted to night shifts indi- } \\
\text { cated by a delay of the aMT6s rhythm, two } \\
\text { subjects did not adapt to nights, while one sub- } \\
\text { ject appeared to be adapted to night shift before } \\
\text { the study started. Large individual variations } \\
\text { in the subsequent adaptation to day work; two } \\
\text { subjects continued to phase delay, one subject } \\
\text { showed phase advance, and five subjects did } \\
\text { not change their phase position. }\end{array}$ \\
\hline $\begin{array}{l}\text { Gibbs et al. } \\
(2007)^{43)}\end{array}$ & $\begin{array}{l}\text { Collection of urine samples several } \\
\text { times a day for two weeks of swing } \\
\text { shift (7N/7D). Actigraph worn con- } \\
\text { tinuously. Shift duration was } 12 \mathrm{~h} \text {. }\end{array}$ & $\begin{array}{l}23 \text { male offshore } \\
\text { swing shift workers in } \\
\text { the North Sea, mean } \\
\text { age } 40.2 \mathrm{yr}(\mathrm{UK}) \text {. }\end{array}$ & $\begin{array}{l}\text { Adaptation to } \\
\text { night work and } \\
\text { back to day } \\
\text { work. }\end{array}$ & $\begin{array}{l}\text { Changes in } \\
\text { aMT6s acro- } \\
\text { phase. Actigraph } \\
\text { measuring } \\
\text { activity. }\end{array}$ & $\begin{array}{l}19 \text { of } 23 \text { subjects adapted their aMT } 6 \text { s rhythm } \\
\text { to night shift by delay. Of the } 19 \text { night adaptors } \\
7 \text { showed little phase change, } 6 \text { continued } \\
\text { to phase delay and } 6 \text { phase advanced when } \\
\text { swinging back to day shift. Only the latter } \\
\text { group achieved full re-adaptation within a } \\
\text { week. }\end{array}$ \\
\hline
\end{tabular}


Table 2. continued

\begin{tabular}{|c|c|c|c|c|c|}
\hline Authors/ date & Study method & Sample & Research topic & Measures & Main results \\
\hline $\begin{array}{l}\text { Thorne et al. } \\
(2008)^{24)}\end{array}$ & $\begin{array}{l}\text { Urine samples collected every four } \\
\text { hours during the last three days of } 14 \\
\text { or } 21 \text { days of fixed night shifts. Sleep } \\
\text { diary completed daily. Actigraphy } \\
\text { recordings. Comparison between two } \\
\text { night shift schedules ( } 1800-0600 \text { and } \\
\text { 1900-0700). Shift duration as } 12 \mathrm{~h} .\end{array}$ & $\begin{array}{l}\text { Two groups of male } \\
\text { night shift offshore } \\
\text { personnel, } \mathrm{N}=10 \text {, } \\
\text { mean age } 46 \mathrm{yr} \text {, and } \\
\mathrm{N}=7 \text {, mean age } 41 \mathrm{yr} \\
\text { (UK). }\end{array}$ & $\begin{array}{l}\text { Adaptation to } \\
\text { night work. }\end{array}$ & $\begin{array}{l}\text { Changes } \\
\text { in aMT6s } \\
\text { acrophase. } \\
\text { Subjective and } \\
\text { objective sleep. } \\
\text { Actigraphy to } \\
\text { assess activity. }\end{array}$ & $\begin{array}{l}14 \text { of } 16 \text { night workers showed circadian } \\
\text { adaptation to night shift. Adapted workers } \\
\text { (1900-0700 hours) had significantly later } \\
\text { acrophase assessed by actigraph compared to } \\
\text { adapted subjects working } 1800-0600 \text { hours. }\end{array}$ \\
\hline $\begin{array}{l}\text { Barnes et al. } \\
(1998)^{44)}\end{array}$ & $\begin{array}{l}\text { Urine samples collected several times } \\
\text { a day during two weeks of swing shift } \\
(7 \mathrm{D} / 7 \mathrm{~N}) \text {. Sleep reports completed } \\
\text { daily and wrist activity monitor worn } \\
\text { continuously for two weeks, } 12 \mathrm{~h} \\
\text { shifts. }\end{array}$ & $\begin{array}{l}\text { Two male drill crews } \\
\text { in the North Sea, } 11 \\
\text { studied in November, } \\
\text { mean age } 34.6 \mathrm{yr} \text { and } \\
7 \text { studied in March, } \\
\text { mean age } 32.6 \mathrm{yr} \\
\text { (UK). }\end{array}$ & $\begin{array}{l}\text { Adaptation to } \\
\text { night work. }\end{array}$ & $\begin{array}{l}\text { Changes in } \\
\text { aMT63- acro- } \\
\text { phase. Sleep } \\
\text { during work } \\
\text { period. }\end{array}$ & $\begin{array}{l}\text { The crew studied in November showed no } \\
\text { change in aMT6s rhythm during night shift, } \\
\text { while the crew studied in March showed a } \\
\text { significant phase advance of the rhythm during } \\
\text { night shift. Sleep duration was significantly } \\
\text { shorter on night shift in November. Season } \\
\text { may affect adaptation. }\end{array}$ \\
\hline $\begin{array}{l}\text { Harris et al. } \\
(2010)^{29)}\end{array}$ & $\begin{array}{l}\text { Offshore workers followed during } \\
\text { three two-week work periods (14D, } \\
\text { 14N, 7N/7D) They were monitored } \\
\text { for four weeks; one before, two dur- } \\
\text { ing and one after the shift work. Shift } \\
\text { duration was } 12 \mathrm{~h} \text {. }\end{array}$ & $\begin{array}{l}19 \text { oil rig workers } \\
\text { in the North Sea. } 6 \\
\text { females, } 13 \text { males. } \\
\text { Mean age } 44.4 \text { yr. } \\
\text { Response rate } 87.5 \% \text {. } \\
\text { Sample as for Waage } \\
\text { et al. (2012) and } \\
\text { Saksvik et al. (2011) } \\
\text { (Norway). }\end{array}$ & $\begin{array}{l}\text { Effects of } \\
\text { changing from } \\
\text { fixed day/night } \\
\text { shifts to } 7 \mathrm{~N} / 7 \mathrm{D} \\
\text { swing shifts in } \\
\text { terms of cortisol } \\
\text { rhythm, reaction } \\
\text { time and health. } \\
\text { Adaptation and } \\
\text { re-adaptation. }\end{array}$ & $\begin{array}{l}\text { Cortisol assessed } \\
\text { via saliva } 5 \\
\text { times each test } \\
\text { day ( } 3 \text { or } 4 \\
\text { days during } \\
2 \text { weeks at } \\
\text { work). Reaction } \\
\text { time assessed } \\
\text { at start and at } \\
\text { end of each } \\
\text { shift. Health } \\
\text { complaints and } \\
\text { perception of } \\
\text { work assessed at } \\
\text { baseline (fixed } \\
\text { shift) and when } \\
\text { working swing } \\
\text { shift (7N/7D). }\end{array}$ & $\begin{array}{l}\text { Swing shift gave no negative health effects or } \\
\text { negative changes in reaction time during the } \\
\text { day they shifted from night work to day work. } \\
\text { Adaptation to night shift was complete within } \\
\text { a week regardless of schedule, while recovery } \\
\text { from night shift took a longer time. Follow- } \\
\text { ing swing shift, the cortisol rhythms were } \\
\text { readapted one week after the work period. The } \\
\text { rhythms were not readapted after one week at } \\
\text { home following fixed night shift. }\end{array}$ \\
\hline $\begin{array}{l}\text { Bjorvatn et al. } \\
(1998)^{45)}\end{array}$ & $\begin{array}{l}\text { Sleep diary completed daily dur- } \\
\text { ing two weeks of night work and } \\
\text { during the first week after returning } \\
\text { home. Questionnaire completed after } \\
\text { the night work period and after the } \\
\text { re-adaptation period at home. Shift } \\
\text { duration was } 12 \mathrm{~h} \text {. }\end{array}$ & $\begin{array}{l}7 \text { male oil platform } \\
\text { workers in the North } \\
\text { Sea, mean age } 38.9 \\
\text { yr. Sample as for } \\
\text { Bjorvatn et al. (1999) } \\
\text { (Norway). }\end{array}$ & $\begin{array}{l}\text { Adaptation to } \\
\text { night work and } \\
\text { daytime sched- } \\
\text { ule at home. }\end{array}$ & $\begin{array}{l}\text { Subjective sleep } \\
\text { and sleepiness. }\end{array}$ & $\begin{array}{l}\text { Adaptation to night work was complete within } \\
\text { a few days, indicated by rapid reduction in } \\
\text { sleepiness. Re-adaptation to daytime schedule } \\
\text { at home was slower and more difficult. Subjec- } \\
\text { tive sleepiness was higher during the week at } \\
\text { home, compared to the two weeks working } \\
\text { nights. }\end{array}$ \\
\hline $\begin{array}{l}\text { Bjorvatn et al. } \\
(2006)^{46)}\end{array}$ & $\begin{array}{l}\text { Placebo group in an RCT. Serial } \\
\text { reaction time tests at } 3 \text { time points } \\
\text { during } 3 \text { nights/day of each week of a } \\
\text { two week swing shift period ( } 7 \mathrm{~N} / 7 \mathrm{D}) \text {, } \\
\text { daily sleep diary, actigraphy monitor- } \\
\text { ing, and questionnaire administered } \\
\text { after the work period. Shift duration } \\
\text { was } 12 \mathrm{~h} \text {. }\end{array}$ & $\begin{array}{l}17 \text { offshore drill- } \\
\text { ing rig personnel in } \\
\text { the North Sea with } \\
\text { self-reported shift } \\
\text { work adjustment } \\
\text { problems. Mean age } \\
42 \text { yr. One female, } 16 \\
\text { males. Sample as for } \\
\text { Bjorvatn } \text { et al. (2007) } \\
\text { (Norway). }\end{array}$ & $\begin{array}{l}\text { Adaptation to } \\
\text { night work and } \\
\text { re-adaptation to } \\
\text { day work. }\end{array}$ & $\begin{array}{l}\text { Sleep and sleepi- } \\
\text { ness. Perceived } \\
\text { adaptation and } \\
\text { re-adaptation. }\end{array}$ & $\begin{array}{l}\text { Subjective and objective measures of sleep and } \\
\text { sleepiness gradually improved during night } \\
\text { work. The return to day work after } 1 \text { week of } \\
\text { night shift caused an increase in subjective } \\
\text { sleepiness and worsening of sleep parameters. } \\
\text { Sleep and sleepiness gradually improved dur- } \\
\text { ing the week of day work. }\end{array}$ \\
\hline $\begin{array}{l}\text { Bjorvatn et al. } \\
(1999)^{47)}\end{array}$ & $\begin{array}{l}\text { Baseline (control), thereafter one } \\
\text { condition with bright light treatment } \\
\text { administered the first four nights of } \\
14 \text { night shift and the first } 4 \text { days at } \\
\text { home, scheduled individually to phase } \\
\text { delay the circadian rhythm. Daily data } \\
\text { collection during two weeks of night } \\
\text { work offshore and one week at home, } \\
\text { using sleep diary. Questionnaires } \\
\text { were answered following shift work } \\
\text { and following re-adaptation period at } \\
\text { home. Shift duration was } 12 \mathrm{~h} \text {. }\end{array}$ & $\begin{array}{l}7 \text { male night shift } \\
\text { workers in the North } \\
\text { Sea, mean age } 38.9 \\
\text { yr. Sample as for } \\
\text { Bjorvatn et al. (1998) } \\
\text { (Norway). }\end{array}$ & $\begin{array}{l}\text { Adaptation to } \\
\text { night work and } \\
\text { daytime sched- } \\
\text { ule at home. }\end{array}$ & $\begin{array}{l}\text { Self-reported } \\
\text { sleep and sleepi- } \\
\text { ness. } \\
\text { Subjective } \\
\text { adaptation and } \\
\text { re-adaptation. }\end{array}$ & $\begin{array}{l}\text { No significant effect of light treatment was } \\
\text { found during night shifts. Bright light treatment } \\
\text { at home led to significantly better self-reported } \\
\text { sleep and reduced sleepiness compared to the } \\
\text { control condition, and improved also daytime } \\
\text { functioning. }\end{array}$ \\
\hline
\end{tabular}


Table 2. continued

\begin{tabular}{|c|c|c|c|c|c|}
\hline Authors/ date & Study method & Sample & Research topic & Measures & Main results \\
\hline $\begin{array}{l}\text { Bjorvatn et al. } \\
(2007)^{48)}\end{array}$ & $\begin{array}{l}\text { Randomized placebo-controlled, } \\
\text { crossover study. Participants received } \\
\text { placebo, melatonin or bright light } \\
\text { treatment during the first four days of } \\
\text { night shift or day shift. The workers } \\
\text { worked swing shift during all condi- } \\
\text { tions (7N/7D), } 12 \text { h shifts. }\end{array}$ & $\begin{array}{l}17 \text { offshore drill- } \\
\text { ing rig personnel in } \\
\text { the North Sea with } \\
\text { self-reported shift } \\
\text { work adjustment } \\
\text { problems. Mean age } \\
42 \text { yr. One female, } 16 \\
\text { males. Sample as for } \\
\text { Bjorvatn et al. (2006) } \\
\text { (Norway). }\end{array}$ & $\begin{array}{l}\text { Effects of mela- } \\
\text { tonin and bright } \\
\text { light treatment } \\
\text { on adaptation to } \\
\text { night work. }\end{array}$ & $\begin{array}{l}\text { Daily sleep } \\
\text { diary comple- } \\
\text { tion, actigraphy } \\
\text { monitoring, } \\
\text { serial reaction } \\
\text { time tests and } \\
\text { questionnaires. }\end{array}$ & $\begin{array}{l}\text { Sleep diary and questionnaire showed that } \\
\text { melatonin reduced sleepiness, increased } \\
\text { sleep duration, improved sleep efficiency and } \\
\text { improved the "quality of the day" during day } \\
\text { shift. Bright light treatment gave a modest } \\
\text { reduction in sleep onset latency during night } \\
\text { shift, compared to placebo and melatonin. } \\
\text { Melatonin reduced the number of days needed } \\
\text { for re-adaptation compared to bright light, } \\
\text { while the placebo condition reached an inter- } \\
\text { mediate level. Actigraph showed that melato- } \\
\text { nin increased sleep onset latency compared to } \\
\text { bright light and placebo during day work. }\end{array}$ \\
\hline $\begin{array}{l}\text { Thorne et al. } \\
(2010)^{49)}\end{array}$ & $\begin{array}{l}\text { Randomized crossover design, } \\
\text { subjects assessed for } 21 \text { days; the last } \\
\text { week of two or three weeks of night } \\
\text { shift work and the following two } \\
\text { weeks at home. In one condition the } \\
\text { subjects wore sunglasses from wake- } \\
\text { up until they received light treatment } \\
\text { beginning at } 1300 \mathrm{~h} \text { the first day, on } \\
\text { subsequent days beginning earlier. In } \\
\text { the other condition no intervention } \\
\text { was given. Shift duration was } 12 \mathrm{~h} \text {. }\end{array}$ & $\begin{array}{l}\text { Male offshore night } \\
\text { shift workers in the } \\
\text { North Sea, } \mathrm{N}=10 \text {, } \\
9 \text { or } 8 \text { (for different } \\
\text { measures), mean age } \\
46 \mathrm{yr} \text { and } 49 \mathrm{yr} \text { (UK). }\end{array}$ & $\begin{array}{l}\text { Effects of light } \\
\text { treatment on } \\
\text { sleep and adap- } \\
\text { tation from night } \\
\text { work to daytime } \\
\text { schedule at } \\
\text { home. }\end{array}$ & $\begin{array}{l}\text { Questionnaires, } \\
\text { daily sleep } \\
\text { diary comple- } \\
\text { tion, actigraphy } \\
\text { recordings, } \\
\text { urine samples } \\
\text { collected several } \\
\text { times a day for } \\
\text { three weeks. }\end{array}$ & $\begin{array}{l}\text { As measured by actigraph, sleep efficiency } \\
\text { was improved during light treatment work } \\
\text { period. After the light treatment, sleep onset } \\
\text { was significantly earlier and sleep duration was } \\
\text { significantly longer. Subjective data showed a } \\
\text { decrease in sleep quality in the light treatment } \\
\text { condition. aMT6s measures showed no effect } \\
\text { of light on circadian adaptation. }\end{array}$ \\
\hline $\begin{array}{l}\text { Lauridsen } \\
\text { \& Tønnesen } \\
(1990)^{54)}\end{array}$ & $\begin{array}{l}\text { Analysis of drilling injuries in Norwe- } \\
\text { gian offshore oil production. }\end{array}$ & $\begin{array}{l}\text { Information regarding } \\
3,200 \text { injuries in the } \\
\text { period } 1980-1987, \\
\text { retrieved from the } \\
\text { Rogaland Research } \\
\text { Institute's data base } \\
\text { "Injuries in Offshore } \\
\text { Drilling" (Norway). }\end{array}$ & Injuries. & Injury rates. & $\begin{array}{l}\text { There was no difference between day and night } \\
\text { shifts in terms of injury rate among active } \\
\text { drilling crews. The was a significant increase } \\
\text { in number of injuries between } 0000 \text { and } 0600 \\
\text { hours, as compared to between } 1800 \text { and } 2400 \\
\text { hours. }\end{array}$ \\
\hline
\end{tabular}

$\mathrm{D}=$ day $(\mathrm{s}) ; \mathrm{N}=$ night $(\mathrm{s}) ;$ aMT6s = 6-sulphatoxymelatonin $; \mathrm{RCT}=$ randomized controlled trial

aMT6s acrophase showed no evidence of faster adaptation in the treatment condition. One of the abovementioned intervention studies also assessed the effect of melatonin administration on adaptation, which was found to be marginally more effective than the light treatment ${ }^{48)}$.

Overall, the three intervention studies indicated that bright light treatment and melatonin administration aiming to improve adaptation to shift work offshore may give significant, albeit small, positive effects ${ }^{47-49)}$. The interventions seemed to be more effective in improving readaptation following night work.

\section{Shift work and health}

Six cross-sectional studies examined the relationship between shift work and health.

One study demonstrated that the offshore workers had significantly higher anxiety scores than onshore workers across day work, night work and the off work period ${ }^{32)}$. In two separate studies, day/night workers reported higher scores of psychological distress than day workers ${ }^{33,50)}$. However, the effect of shift work in predicting higher mental distress disappeared in both studies when controlling for individual factors or job type ${ }^{33,50)}$.

Two studies regarding body mass index (BMI) have compared offshore day workers with offshore day/night workers. In one of these, continued exposure to day/night shift work was associated with increased $\mathrm{BMI}^{51)}$, while in the other study day/night shift workers had significantly lower BMI scores than day workers ${ }^{33)}$. The latter study also demonstrated that shift work predicted gastric problems ${ }^{33)}$. A separate study found no differences between offshore shift workers and onshore workers in somatic symptoms such as tiredness and headaches, or in social dysfunction $^{32)}$. Two studies compared offshore workers across different shift schedules; one found no association between shift type or shift work exposure and subjective health complaints ${ }^{34)}$, while in the other, no differences were found regarding subjective health complaints between shift workers without SWD and day workers ${ }^{6}$. In the latter study, subjects with SWD reported more subjective health complaints than individuals without $\mathrm{SWD}^{6}$.

One longitudinal study assessing subjective health 
complaints found no differences between fixed shifts and swing shifts ${ }^{29)}$, similar to another study that found no differences in subjective health complaints between day workers and swing shift workers ${ }^{40)}$.

Overall, few studies have investigated the relationship between shift work and health offshore, and their findings are inconclusive ${ }^{32,33,50)}$. The findings regarding BMI are also inconsistent ${ }^{33,51)}$. Further, it appears that shift work is a predictor for gastric problems ${ }^{33)}$. There is no evidence for more subjective health complaints among offshore shift workers compared to offshore day workers, except in the group of shift workers fulfilling the criteria for $\mathrm{SWD}^{6}$.

\section{Shift work and family/social life}

Two cross-sectional studies examined the impact of shift work on social and family life. A qualitative study with no contrast group found that shift workers offshore had difficulties in reconciling work with family life ${ }^{35)}$. In the other study, the majority of shift workers across all shift schedules reported few problems with social and family life caused by shift work, and in this regard offshore workers were better off than their onshore counterparts ${ }^{52)}$. In sum, the results from these two studies are inconsistent regarding whether offshore shift work is related to problems with family and social life.

\section{Shift work and accidents/safety}

One study demonstrated that with no control for job type, shift work predicted work-related injuries ${ }^{33)}$. In another study, the accident registry for an offshore unit was examined, finding that 17 accidents were recorded during the past year ${ }^{35}$. Nine accidents took place between 0600 and 1800 hours, while eight happened between 1801 and 0559 hours. Taking into account the uneven distribution of workers at night /daytime, they calculated that the relative risk of accidents was $51 \%$ higher during the dark period between 1801 and 0559 hours ${ }^{35)}$. Further, one study analyzed information regarding 3200 drilling injuries in the Norwegian offshore oil production between 1980 and 1987, and found that there was no difference between day and night shifts in the injury rate among active drilling crews $^{53)}$.

Four studies have used serial reaction time tests in order to obtain objective measures of sleepiness and performance. Reaction time measures may be relevant for accidents and safety. Findings from three longitudinal studies demonstrated that, with one exception, there were no significant differences in reaction time across different shift schedules $^{29,39,46)}$. Further, two of the studies found a reduction in reaction time across days for the night shift ${ }^{39,46)}$. Also, a small, but significantly higher mean score in reaction time the first night offshore, when on night shift compared to day shift, was found ${ }^{29)}$. In an intervention study, no significant differences across night shift and day shift in terms of reaction time were found ${ }^{48)}$. During day shift however, this study demonstrated an increased reaction time after intake of melatonin and exposure to bright light during the first day after swinging from night to day work ${ }^{48)}$.

In total, two studies regarding accidents and safety have demonstrated that shift work increased the risk of work-related accidents, and that the relative risk of accidents was higher during the night than day ${ }^{33}{ }^{35}$ ). One study reported no differences in injury rates between day and nighttime in active drilling crews ${ }^{53)}$. The findings from reaction time tests gave no indication that reaction time is longer during night shift work ${ }^{29,39,46)}$.

Shift work, work perceptions and perceived mastery of work

One study comparing the work perceptions (referring to perceived work environment measures, including physical stressors, job demand, job control, skill discretion, supervisor support, and safety perceptions) among day and shift workers across onshore and offshore settings showed that differences between day and shift workers were less marked in the offshore setting; the only significant difference offshore was that shift workers reported significantly greater exposure to physical environmental stressors than day workers ${ }^{30)}$.

Another study investigated factors related to perceived mastery of work (referring to an individual's perception of the desirability of his/her effort at work), and found that night work was unrelated to perceived mastery of work in a multivariate analysis ${ }^{54)}$. In the same study it was shown that offshore workers reported higher levels of perceived mastery of work than a control group.

Overall, offshore shift workers reported greater exposure to physical environmental stressors than offshore day workers ${ }^{30)}$. Night work seems to be unrelated to perceived mastery of work among offshore workers ${ }^{54)}$.

\section{Discussion}

In the present review we have explored studies concerning effects of shift and night work on employees in the offshore petroleum industry. Twenty-nine studies with outcome measures in terms of sleep, adaptation, health, family- and social life, safety, and work perceptions were 
included.

The findings concerning sleep parameters such as quality and duration of sleep are somewhat inconsistent regarding whether day or night workers are better off ${ }^{28}, 31,34,36,37$. However, the majority of studies found that offshore day shift workers reported better sleep quality, longer sleep duration and fewer sleep problems such as difficulty falling and staying asleep, and fragmented sleep, than offshore night shift workers ${ }^{28,31,33,37)}$. These findings are in line with research conducted on shift workers onshore, where shift work has been found to be associated with difficulties falling asleep, shortened sleep duration, and increased fatigue $^{4)}$. The SWD prevalence of $23.3 \%$ obtained in the offshore study is midway between the prevalence in studies from onshore settings ${ }^{6-8)}$. Inconsistent findings may be related to methodological differences between the conducted studies. Some studies have employed sleep and sleepiness measures exclusively based on self-reports, such as questionnaires, sleep diaries and interviews, while others have used objective measures of sleep such as actigraphy. These methodological differences may yield differing results, as research suggests that responses to sleep questionnaires only are moderately correlated with actigraph-measured sleep ${ }^{55)}$.

The main findings regarding adaptation of circadian rhythms were that the most workers were fully adapted to night work within one $\left.{ }^{29}, 39,41-43,45,46\right)$ to two or three weeks ${ }^{24)}$. It appears that full adaptation to night work offshore may be more common and easier to achieve than adaptation to night work onshore, as only a small minority of onshore night workers evidenced complete adaptation ${ }^{9)}$. This may be related to the fact that the offshore environment is better adapted to 24 hour operations with similar routines for day and night shift workers, making it easier to entrain the circadian rhythm to the working environment. Morning exposure to light may prevent a phase delay ${ }^{56)}$. Little exposure to morning light in night workers in the offshore petroleum industry has been suggested as a major reason for faster adaptation offshore ${ }^{45}$. Complete adaptation to night work is beneficial when working nights. However, there is also a disadvantage with the complete adaptation, in that re-adaptation back to a daytime schedule may become more difficult. Several studies in this review showed that re-adaptation from night work back to a daytime schedule offshore or at home was slower than adaptation to night work ${ }^{29,42,45)}$. Given that adaptation to night work is easier offshore, one might predict that re-adaptation back to a daytime schedule is more difficult offshore than onshore, which is in fact what most studies in the present review indicated. Several different methods have been used to measure adaptation to and re-adaptation from night work (e.g., hormones such as aMT6s and cortisol, actigraphy and sleep/sleepiness questionnaires and diaries, as well as reaction time tests). Yet, the findings from studies in this review indicate that the results are largely consistent across measurement methods.

The three intervention studies indicated that specific interventions aiming to improve adaptation offshore may give small albeit significant, positive effects, whereas interventions seemed to be more effective in improving readaptation following night work ${ }^{47-49)}$. This is in line with previous findings from both simulated night work studies and field studies, suggesting that bright light interventions and melatonin administration may improve adaptation to night work - for a review, see Pallesen et al. ${ }^{57)}$.

Few studies have investigated the relationship between shift work and health offshore. Findings from studies conducted onshore indicate that shift work is a risk factor for poor mental health ${ }^{18,19)}$, however, findings from the offshore petroleum industry are somewhat more inconclusive $^{32,33,50)}$. The findings from offshore studies regarding BMI are inconsistent ${ }^{33,51)}$, and do not exclusively support the findings from onshore studies with shift work being a risk factor for increased BMI. Further, it appears that shift work is a predictor for gastric problems ${ }^{33)}$, an association that has also been found in studies onshore ${ }^{12)}$. There is no evidence for more subjective health complaints among offshore shift workers compared to day workers, except in the group of offshore shift workers fulfilling the criteria for $\mathrm{SWD}^{6}$. Overall, these studies are too few in number to justify general conclusions about health-related effects of shift work. In onshore studies, night work seems to be a risk factor for breast cancer among women, however, none of the studies included in this review concern risk for cancer. Neither has any studies regarding reproductive health been conducted. More research regarding health effects of offshore shift work is therefore warranted. Also, it should be noted that the studies in this review mainly or exclusively include male subjects.

Only two studies concerning family and social life have been conducted ${ }^{35,52)}$, finding inconsistencies in whether shift work is associated with problems with family and social life. One of these studies ${ }^{35)}$ supports the finding from onshore research that working shift may be associated with conflict between work and family/social life ${ }^{22}$.

Regarding accidents and safety, two studies have demonstrated that shift work increased the risk of workrelated accidents, and that the relative risk of accidents is 
higher during the night ${ }^{33,35)}$. This is in line with research conducted onshore, where shift and night work have been shown to increase the risk for work-related accidents ${ }^{10)}$. However, one study reported no differences in injury rates between day and nighttime in active drilling crews ${ }^{53)}$. The findings from reaction time tests gave no indication that reaction time is longer during night shift.

Overall, it appears that shift work and night work offshore are more problematic than day work offshore. This is largely consistent with findings from onshore settings ${ }^{58)}$. Further, compared to onshore shift workers, offshore shift workers do not seem to be much worse off. This may be somewhat surprising, as the working conditions for offshore workers are assumed to be harsher than those of the onshore workers. However, the findings may be related to the fact that offshore workers constitute a highly selected and healthy population as the oil industry has strict regulations in terms of health and medication use, and all personnel have regularly health checks. Thus, the offshore workers may be better able to handle the different and perhaps worse working conditions, and it may therefore appear as if the effects of shift work offshore are not particularly worse than shift work onshore. It should also be noted that the offshore work environment (e.g., no commuting time, working mainly indoors and no domestic obligations) may facilitate adaptation to night work to a larger extent than onshore work environment ${ }^{38)}$.

\section{Limitations}

Some limitations of the present review should be noted. Firstly, the results might be affected by publication bias, as only published research was included. Research reports were also excluded due to the uncertainty of their scientific quality, as they have not been peer-reviewed; hence relevant findings may have been lost. Second, only studies written in English were included. It should be noted that some of the articles based on longitudinal data are not independent from each other, as some of them are derived from the same studies (e.g. ${ }^{45,47)}$ ).

There are currently several limitations in the research field that should be taken into consideration when interpreting the results. In general, among studies investigating effects of shift and night work in the offshore petroleum industry, relatively few have been published in peerreviewed journals. Few longitudinal studies on other outcome measures than adaptation and sleep exist. Evidence from the onshore petroleum industry demonstrates that shift work is related to a wide range of negative health consequences, however, few cross-sectional and no longitudinal studies on this relation have been conducted offshore, e.g. only two studies examine BMI changes and none have assessed cancer, cardiovascular health or reproductive health. Further, research on accidents related to shift work offshore is scarce, as is that on its relation to family and social life. Also, the studies concerning health, accidents, family and social life, work perceptions, and some studies concerning sleep, have been cross-sectional in nature; and one can therefore not draw any conclusions about cause and effect from these studies. The methodological design varies greatly across studies, making direct comparison of findings difficult. The category of shift workers includes both those working fixed night shifts and those working swing shift. Additionally, the comparison groups across the different studies vary. In general, it is difficult to find adequate reference groups for these studies. In six of the 29 studies included in this review, offshore shift workers are compared to onshore workers ${ }^{30,32,36,37,52,54)}$. In eight studies, offshore shift workers are compared to offshore day workers $6,28,31,33,34,40,50,51)$, one study compared two different groups of night shift workers ${ }^{24)}$ while in 12 studies, the same offshore workers are compared across different shifts $\left.{ }^{29}, 38,39,41-49\right)$. Two of the studies have no comparison group at all, i.e. the study based on qualitative interviews ${ }^{35)}$ and the study of analysis of drilling injuries ${ }^{53)}$. An additional issue is the various definitions of shift work used across studies ${ }^{59)}$. These methodological issues support the notion that one should be cautious when trying to draw general conclusions from these studies.

As previously mentioned, offshore employees constitute a selected and healthy population. Thus they probably differ from shift workers onshore, and this complicates comparison of results between offshore and onshore settings. The latter may also make these kinds of comparisons less informative, and may suggest that comparisons across groups of offshore workers (e.g., day vs. night workers) are more informative.

The number of participants across the offshore studies varies greatly from $7^{45,47)}$ to $9,601^{31)}$. Non-significant findings may in some of the smallest studies reflect lack of statistical power, rather than absence of relationships ${ }^{60)}$.

The vast majority of studies derive from Europe; more specifically, 27 of the studies stem from the Norwegian and the UK offshore sector. Only two of the studies have been conducted outside Europe (Brazil). A large proportion of the worldwide offshore oil and gas industry is situated in the North Sea, and it is therefore rather natural that most studies derive from this area. More research conducted outside of Europe and the North Sea is still 
warranted in the future.

\section{Implications for future research}

Generally, the relatively few studies conducted and the inconsistent findings in the field give rise to a need for more research about the effects of shift work in the offshore petroleum industry. More longitudinal research would be beneficial in order to investigate long-term effects of working offshore in the petroleum industry, but also to investigate day-to-day variations on outcome variables over different shifts to establish short-term changes in e.g. health, sleep and well-being. This need is especially pronounced concerning health effects, as there is a lack of longitudinal studies concerning health in offshore shift workers. Future offshore shift work studies should aim at including objectively measured metabolic and cardiovascular parameters. Studies concerning the risk of cancer and gastrointestinal disorders/symptoms as a consequence of shift work, preferably assessed by objective measures, should also be prioritized in future offshore studies. Further, as the geographical areas covered by the present studies are rather restricted, research from other parts of the world is warranted. Another gap in current knowledge regards individual factors that may influence adaptation to and also effects of shift work offshore. As research from work settings onshore indicates that personality variables such as neuroticism, extraversion, morningness, hardiness, flexibility and languidity influence the tolerance to shift work $^{61)}$, future studies should focus upon this issue.

\section{Conclusion}

The longitudinal studies were generally consistent in showing that adaptation to night work was complete within one to two weeks of work, while re-adaptation to a daytime schedule was slower. Shift workers reported more sleep problems than day workers. The data regarding mental and physical health, family and social life, and accidents yielded inconsistent results, and were insufficient as a base for drawing general conclusions. More research in the field is warranted.

\section{References}

1) Monk TH, Folkard S (1992) Making shift work tolerable. Taylor \& Francis, London.

2) Åkerstedt T (1998) Shift work and disturbed sleep/ wakefulness. Sleep Med Rev 2, 117-28. [Medline] [CrossRef]
3) Costa G, Haus E, Stevens R (2010) Shift work and cancerconsiderations on rationale, mechanisms, and epidemiology. Scand J Work Environ Health 36, 163-79. [Medline] [CrossRef]

4) Åkerstedt T (2003) Shift work and disturbed sleep/ wakefulness. Occup Med (Lond) 53, 89-94. [Medline] [CrossRef]

5) American Academy of Sleep Medicine (2005) International Classification of Sleep Disorders, 2nd Ed. In: Diagnostic and Coding Manual. American Academy of Sleep Medicine, Westchester.

6) Waage S, Moen BE, Pallesen S, Eriksen HR, Ursin H, Åkerstedt T, Bjorvatn B (2009) Shift work disorder among oil rig workers in the North Sea. Sleep 32, 558-65. [Medline]

7) Drake CL, Roehrs T, Richardson G, Walsh JK, Roth T (2004) Shift work sleep disorder: prevalence and consequences beyond that of symptomatic day workers. Sleep 27, 1453-62. [Medline]

8) Flo E, Pallesen S, Magerøy N, Moen BE, Grønli J, Nordhus IH, Bjorvatn B (2012) Shift work disorder in nursesassessment, prevalence and related health problems. PLoS ONE 7, e33981. [Medline] [CrossRef]

9) Folkard S (2008) Do permanent night workers show circadian adjustment? A review based on the endogenous melatonin rhythm. Chronobiol Int 25, 215-24. [Medline] [CrossRef]

10) Folkard S, Lombardi DA, Tucker PT (2005) Shiftwork: safety, sleepiness and sleep. Ind Health 43, 20-3. [Medline] [CrossRef]

11) Bøggild H, Knutsson A (1999) Shift work, risk factors and cardiovascular disease. Scand J Work Environ Health 25, 85-99. [Medline] [CrossRef]

12) Knutsson A, Bøggild H (2010) Gastrointestinal disorder among shift workers. Scand J Work Environ Health 36, 85-95. [Medline] [CrossRef]

13) Megdal SP, Kroenke CH, Laden F, Pukkala E, Schernhammer ES (2005) Night work and breast cancer risk: a systematic review and meta-analysis. Eur J Cancer 41, 2023-32. [Medline] [CrossRef]

14) Schernhammer ES, Kroenke $\mathrm{CH}$, Laden F, Hankinson SE (2006) Night work and risk of breast cancer. Epidemiology 17, 108-11. [Medline] [CrossRef]

15) Karlsson B, Knutsson A, Lindahl B (2001) Is there an association between shift work and having a metabolic syndrome? Results from a population based study of 27 485 people. Occup Environ Med 58, 747-52. [Medline] [CrossRef]

16) Karlsson $\mathrm{BH}$, Knutsson $\mathrm{AK}$, Lindahl BO, Alfredsson LS (2003) Metabolic disturbances in male workers with rotating three-shift work. Results of the WOLF study. Int Arch Occup Environ Health 76, 424-30. [Medline] [CrossRef]

17) Morikawa $Y$, Nakagawa $H$, Miura $K$, Soyama $Y$, Ishizaki $M$, Kido T, Naruse Y, Suwazono Y, Nogawa K (2007) Effect of shift work on body mass index and metabolic parameters. 
Scand J Work Environ Health 33, 45-50. [Medline] [CrossRef]

18) Bildt C, Michélsen H (2002) Gender differences in the effects from working conditions on mental health: a 4-year follow-up. Int Arch Occup Environ Health 75, 252-8. [Medline] [CrossRef]

19) Bara AC, Arber S (2009) Working shifts and mental health -findings from the British Household Panel Survey (19952005). Scand J Work Environ Health 35, 361-7. [Medline] [CrossRef]

20) Nurminen $T$ (1998) Shift work and reproductive health. Scand J Work Environ Health 24 (suppl 3), 28-34. [Medline]

21) Bonzini M, Palmer KT, Coggon D, Carugno M, Cromi A, Ferrario MM (2011) Shift work and pregnancy outcomes: a systematic review with meta-analysis of currently available epidemiological studies. BJOG 118, 1429-37. [Medline] [CrossRef]

22) Staines GL, Pleck JH (1984) Nonstandard work schedules and family life. J Appl Psychol 69, 515-23. [CrossRef]

23) Petroleumstilsynet [Internet]. RNNP - Spørreskjemaundersøkelsen 2011. Updated 2012 April 25. http://www. ptil.no/nyheter/rnnpspoerreskjemaundersoekelsen2011-article8492-24.html. Accessed September 11, 2012.

24) Thorne H, Hampton S, Morgan L, Skene DJ, Arendt J (2008) Differences in sleep, light, and circadian phase in offshore 18:00-06:00 $\mathrm{h}$ and 19:00-07:00 h shift workers. Chronobiol Int 25, 225-35. [Medline] [CrossRef]

25) Ross JK (2009) Offshore industry shift work - health and social considerations. Occup Med (Lond) 59, 310-5. [Medline] [CrossRef]

26) Mikkelsen A, Ringstad AJ, Steineke JM (2004) Working time arrangements and safety for offshore workers in the North Sea. Saf Sci 42, 167-84. [CrossRef]

27) Parkes KR (2012) Shift schedules on North Sea oil/ gas installations: a systematic review of their impact on performance, safety and health. Saf Sci 50, 1636-51. [CrossRef]

28) Menezes MCR, Pires MLN, Benedito-Silva AA, Tufik S (2004) Sleep parameters among offshore workers: an initial assessment in the Campos Basin, Rio De Janeiro, Brazil. Chronobiol Int 21, 889-97. [Medline] [CrossRef]

29) Harris A, Waage S, Ursin H, Hansen $\AA$ M, Bjorvatn B, Eriksen HR (2010) Cortisol, reaction time test and health among offshore shift workers. Psychoneuroendocrinology 35, 1339-47. [Medline] [CrossRef]

30) Parkes KR (2003) Shiftwork and environment as interactive predictors of work perceptions. J Occup Health Psychol 8, 266-81. [Medline] [CrossRef]

31) Hope S, Øverland S, Brun W, Matthiesen SB (2010) Associations between sleep, risk and safety climate: a study of offshore personnel on the Norwegian continental shelf. Saf Sci 48, 469-77. [CrossRef]

32) Parkes KR (1992) Mental health in the oil industry: a comparative study of onshore and offshore employees. Psychol Med 22, 997-1009. [Medline] [CrossRef]
33) Parkes KR (1999) Shiftwork, job type, and the work environment as joint predictors of health-related outcomes. J Occup Health Psychol 4, 256-68. [Medline] [CrossRef]

34) Waage S, Pallesen S, Moen BE, Bjorvatn B (2010) Shift work and age in petroleum offshore industry. Int Marit Health 62, 251-7. [Medline]

35) Rodrigues VF, Fischer FM, Brito MJ (2001) Shift work at a modern offshore drilling rig. J Hum Ergol (Tokyo) 30, 167-72. [Medline]

36) Parkes KR (1994) Sleep patterns, shiftwork, and individual differences: a comparison of onshore and offshore controlroom operators. Ergonomics 37, 827-44. [Medline] [CrossRef]

37) Parkes KR (2002) Age, smoking, and negative affectivity as predictors of sleep patterns among shiftworkers in two environments. J Occup Health Psychol 7, 156-73. [Medline] [CrossRef]

38) Saksvik IB, Bjorvatn B, Harvey AG, Waage S, Harris A, Pallesen S (2011) Adaptation and readaptation to different shift work schedules measured with sleep diary and actigraphy. J Occup Health Psychol 16, 331-44. [Medline] [CrossRef]

39) Waage S, Harris A, Pallesen S, Saksvik IB, Moen B, Bjorvatn B (2012) Subjective and objective sleepiness among oil rig workers during three different shift schedules. Sleep Med 13, 64-72. [Medline] [CrossRef]

40) Waage S, Pallesen S, Moen BE, Bjorvatn B (2013) Sleep and health in oil rig workers - before and after a two week work period offshore. Ind Health 51, 172-9.

41) Barnes RG, Deacon SJ, Forbes MJ, Arendt J (1998) Adaptation of the 6-sulphatoxymelatonin rhythm in shiftworkers on offshore oil installations during a 2-week 12-h night shift. Neurosci Lett 241, 912.

42) Gibbs M, Hampton S, Morgan L, Arendt J (2002) Adaptation of the circadian rhythm of 6sulphatoxymelatonin to a shift schedule of seven nights followed by seven days in offshore oil installation workers. Neurosci Lett 325, 91-4. [Medline] [CrossRef]

43) Gibbs M, Hampton S, Morgan L, Arendt J (2007) Predicting circadian response to abrupt phase shift: 6-sulphatoxymelatonin rhythms in rotating shift workers offshore. J Biol Rhythms 22, 368-70. [Medline] [CrossRef]

44) Barnes RG, Forbes MJ, Arendt J (1998) Shift type and season affect adaptation of the 6-sulphatoxymelatonin rhythm in offshore oil rig workers. Neurosci Lett 252, 179-82. [Medline] [CrossRef]

45) Bjorvatn B, Kecklund G, Åkerstedt T (1998) Rapid adaptation to night work at an oil platform, but slow readaptation after returning home. J Occup Environ Med 40, 601-8. [Medline] [CrossRef]

46) Bjorvatn B, Stangenes K, Øyane N, Forberg K, Lowden A, Holsten F, Åkerstedt T (2006) Subjective and objective measures of adaptation and readaptation to night work on an oil rig in the North Sea. Sleep 29, 821-9. [Medline]

47) Bjorvatn B, Kecklund G, Åkerstedt T (1999) Bright 
light treatment used for adaptation to night work and readaptation back to day life. A field study at an oil platform in the North Sea. J Sleep Res 8, 105-12. [Medline] [CrossRef]

48) Bjorvatn B, Stangenes K, Øyane N, Forberg K, Lowden A, Holsten F, Åkerstedt T (2007) Randomized placebocontrolled field study of the effects og bright light and melatonin in adaptation to night work. Scand J Work Environ Health 33, 204-14. [Medline] [CrossRef]

49) Thorne HC, Hampton S, Morgan LM, Skene DJ, Arendt J (2010) Returning from night shift to day life: beneficial effects of light on sleep. Sleep Biol Rhythms 8, 212-21. [CrossRef]

50) Ljoså CH, Tyssen R, Lau B (2011) Mental distress among shift workers in Norwegian offshore petroleum industry -relative influence of individual and psychosocial work factors. Scand J Work Environ Health 37, 551-5. [Medline] [CrossRef]

51) Parkes KR (2002) Shift work and age as interactive predictors of body mass index among offshore workers. Scand J Work Environ Health 28, 64-71. [Medline] [CrossRef]

52) Ljoså CH, Lau B (2009) Shiftwork in the Norwegian petroleum industry: overcoming difficulties with family and social life —a cross sectional study. J Occup Med Toxicol 4, 22. [Medline] [CrossRef]

53) Lauridsen $\varnothing$, Tønnesen $T$ (1990) Injuries related to the aspects of shift working: a comparison of different offshore shift arrangements. J Occup Accid 12, 167-76. [CrossRef]

54) Ljoså CH, Tyssen R, Lau B (2013) Perceived mastery of work among shift workers in the Norwegian offshore petroleum industry. Ind health 51, 145-53.

55) Lauderdale DS, Knutson KL, Yan LL, Liu K, Rathouz PJ (2008) Self-reported and measured sleep duration: how similar are they? Epidemiology 19, 838-45. [Medline] [CrossRef]

56) Czeisler CA, Dijk DJ (1995) Use of bright light to treat maladaptation to night shift work and circadian rhythm sleep disorders. J Sleep Res 4, 70-3. [Medline] [CrossRef]

57) Pallesen S, Bjorvatn B, Magerøy N, Saksvik IB, Waage S, Moen BE (2010) Measures to counteract the negative effects of night work. Scand J Work Environ Health 36, 109-20. [Medline] [CrossRef]

58) Flo E, Pallesen S, Åkerstedt T, Magerøy N, Moen BE, Grønli J, Nordhus IH, Bjorvatn B (2013) Shift-related sleep problems vary according to work schedule. Occup Environ Med 70, 238-45. [Medline] [CrossRef]

59) Knutsson A (2004) Methodological aspects of shiftwork research. Chronobiol Int 21, 1037-47. [Medline] [CrossRef]

60) Cohen J (1988) Statistical power analysis for the behavioral sciences, 2nd Ed., Lawrence Erlbaum Associates, Hillsdale.

61) Saksvik IB, Bjorvatn B, Hetland H, Sandal GM, Pallesen S (2011) Individual differences in tolerance to shift work a systematic review. Sleep Med Rev 15, 221-35. [Medline] [CrossRef] 